\title{
Anti-inflammatory properties of Escherichia coli Nissle 1917 in a murine colitis model
}

\author{
Jihye Park $^{1 *}$, Da Hye Kim ${ }^{1,2 *}$, Soochan Kim ${ }^{1,2}$, Hyun Woo Ma ${ }^{1,3}$, I Seul Park ${ }^{1,3}$, Mijeong Son ${ }^{1,3}$, Ji Hyung Kim ${ }^{1,3}$, \\ Yoojin Shin ${ }^{1,3}$, Seung Won Kim ${ }^{1,2,3}$, Jae Hee Cheon ${ }^{1,2,3}$ \\ ${ }^{1}$ Department of Internal Medicine and Institute of Gastroenterology, ${ }^{2}$ Severance Biomedical Science Institute, and ${ }^{3}$ Brain Korea 21 PLUS Project \\ for Medical Science, Yonsei University College of Medicine, Seoul, Korea
}

Escherichia coli Nissle 1917 (EcN) strain is a Gram-negative strain isolated from a young German soldier who were not infected with Shigella during the endemic period at World War I. The probiotic drug Mutaflor ${ }^{\circledR}$ using this EcN is available and used in managing gastrointestinal disorders including diarrhea, uncomplicated diverticular disease, and ulcerative colitis (UC). ${ }^{1}$ As for UC, the relapse rate during 12 months in patients with UC between EcN treated group and mesalazine treated group were statistically equivalent in a randomized, double blind, double dummy trial. ${ }^{1}$ However, there have been few previous studies to demonstrate the mechanism of its probiotic effects. ${ }^{2}$ Also, the anti-inflammatory effects of EcN in mouse colitis models were controversial. ${ }^{3}$ Schultz et al. ${ }^{4}$ reported that there was no effect of administration of $\mathrm{EcN}$ for 10 days on an acute $2 \%$ dextran sulfate sodium (DSS) induced colitis model. On the other hand, Rodríguez-Nogales et al. ${ }^{5}$ demonstrated the effect of EcN administration for 26 days on the acute 3\% DSS-induced colitis model. We aimed this study to investigated the anti-inflammatory properties of EcN in the DSS murine

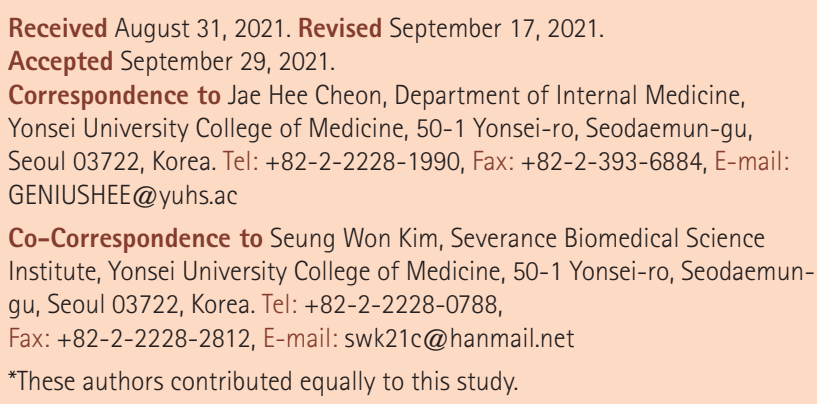

colitis model and evaluated the related inflammatory responses associated with T helper 17 (Th17), Thl, and regulator T (Treg) cells.

Male C57BL/6 mice (8-week-old) were administered 2.5\% (w/v) DSS (MP Biomedicals, Solon, OH, USA) in their drinking water for 6 days, and water was replaced with DSS-free water before euthanization. Animals were randomly assigned to 2 groups; DSS+Veh (phosphate buffered saline [PBS]-treated vehicle) $(n=4)$ and DSS+EcN groups $(n=4)$. The DSS+Veh group was administered with $0.1 \mathrm{~mL}$ PBS, and the DSS+EcN group was administered with $1 \times 10^{8}$ bacteria in $0.1 \mathrm{~mL}$ PBS by oral gavage on days 0,2 , and 5 . All mice were sacrificed 8 days after the onset of the study. Mice were monitored for body weight loss, and the entire colon was isolated from the harvested mouse. The colon length was measured between the ileocecal junction and the rectum. Parts of the distal colon were cut into 2 to 3 pieces for periodic acid-Schiff staining and images were obtained using a model BX41 light microscope (Olympus Optical, Tokyo, Japan). Spleens were harvested for flow cytometry, and cells were analyzed with a FACSVerse flow cytometer (BD Biosciences, San Jose, CA, USA) and were analyzed using FlowJo software (Tree Star, Eugene, OR, USA). ${ }^{6}$ All animal experiments complied with all applicable Korean laws, and were approved by the Institutional Animal Care and Use Committee of Yonsei University Severance Hospital (Seoul, Korea) (IACUC No. 2019-0241). The experiments complied with the approved IACUC guidelines. All results are expressed as standard error of the mean. GraphPad Software (La Jolla, CA, USA) was used for all analyses. The significance of differ- 
ences between conditions was assessed using Mann-Whitney test. $P$-values $<0.05$ were considered significant.

The DSS+EcN group significantly improved body weight compared to the DSS+Veh group $(104.4 \%$ vs. $88.7 \%, P<0.05)$ (Fig. 1A). The colon length was numerically longer in the DSS+ EcN group compared to the DSS+Veh group, although this was not statistically significant $(9.5 \mathrm{~cm}$ vs. $8.6 \mathrm{~cm}, P=0.381$ ) (Fig. 1B). In the histological analysis, histological score was shown to be significantly improved in the DSS $+\mathrm{EcN}$ group compared to DSS+Veh group (3.3 vs. 9.0, $P<0.05$ ) (Fig. 1C and D). Also, goblet cell numbers were significantly increased in the DSS+EcN group compared to DSS+Veh group (2.5 vs. 0.3, $P<0.05$ ) (Fig. 1E). We next investigated T cells in splenocytes by flow cytometry. The number of $\mathrm{CD}^{+} \mathrm{Foxp}^{+}$Treg cell was increased $(P=0.200)$ and that of CD4 $4^{+}$Ror $\gamma t^{+} I L-17^{+}$Th17 cells $(P=0.124)$ were numerically decreased in the DSS+EcN group compared to DSS+Veh group, although this was not statistical- ly significant (Fig. 2).

The several mechanisms of anti-inflammatory properties of $\mathrm{EcN}$ were suggested in the previous studies. EcN modulates the gut barrier by upregulating tight junction proteins including zonula occludens (ZO-1 and ZO-2) and antimicrobial factors including microcins. ${ }^{7}$ Furthermore, host-microbe interactions by dendritic cells could modulate innate and adaptive immunity including T lymphocyte responses. The induction of Tregs by probiotics could lead the new therapeutic approach as an alternative to immunosuppressive drugs. ${ }^{8}$ Weise et al. ${ }^{9}$ reported that EcN induced the $\mathrm{CD} 4^{+} \mathrm{Foxp}^{+}{ }^{+}$Treg cells in an allergen-induced mouse dermatitis model. With regard to mouse colitis model, the mechanism modulating $\gamma \delta$ T cells by EcN was suggested in the previous study, but the impact of Treg/ Th17 responses were not yet evaluated. ${ }^{9}$ We demonstrated the anti-inflammatory properties of $\mathrm{EcN}$ in the DSS-induced murine colitis model. Moreover, our results suggest that EcN may
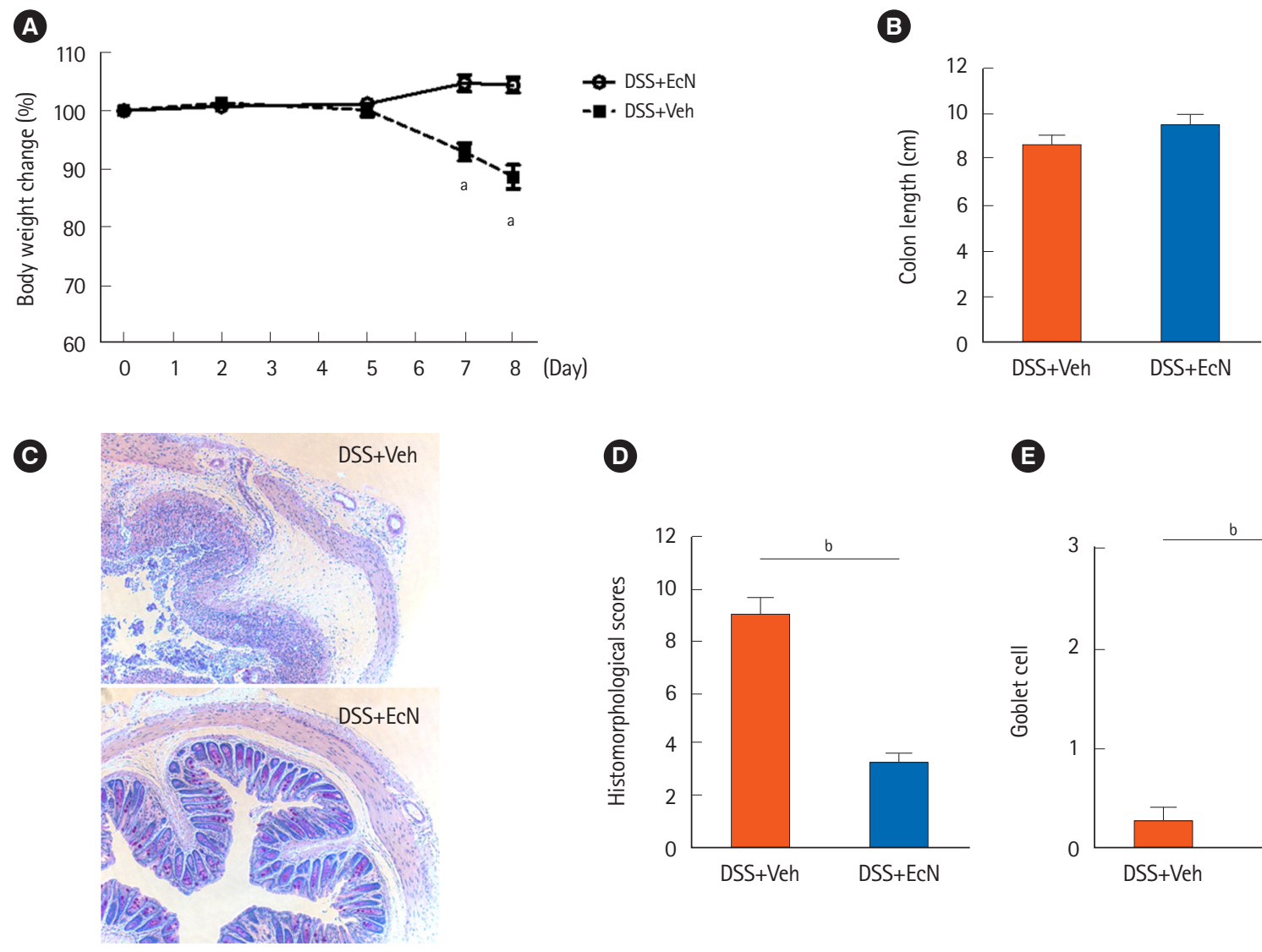

D

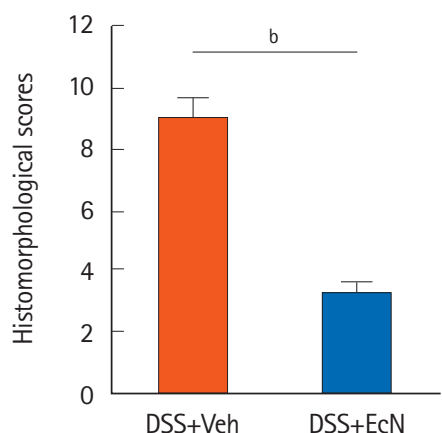

E

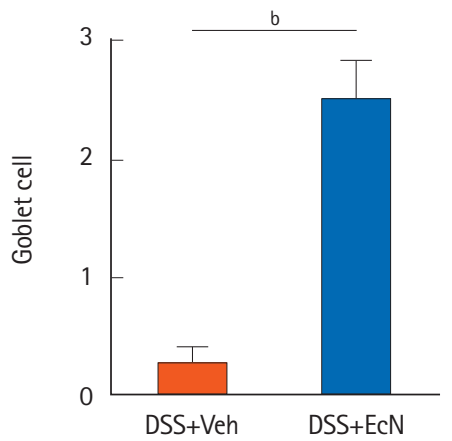

Fig. 1. Escherichia coli Nissle 1917 (EcN) ameliorated dextran sulfate sodium (DSS)-induced colitis. At days 0, 2, and 5, 8-week-old male C57BL/6 mice were administered $1 \times 10^{8} \mathrm{ECN}$ bacteria or phosphate buffered saline daily by gavage (P.0.). 2.5\% DSS was added to the drinking water from day 0 and continued for 6 days. Body weight was recorded throughout the 8 days. (A) All mice were euthanized on day 8 of the study and colon length $(B)$, periodic acid-Schiff staining $(\times 100)(C)$, histomorphological scores $(D)$, and goblet cell score $(E)$ were analyzed. Data are expressed as the mean \pm standard error of the mean $(n=4)$. Analyses were performed using Mann-Whitney test. ${ }^{a} P<0.05$, ${ }^{\mathrm{b}} \mathrm{P}<0.01$. Veh, phosphate buffered saline-treated vehicle. 

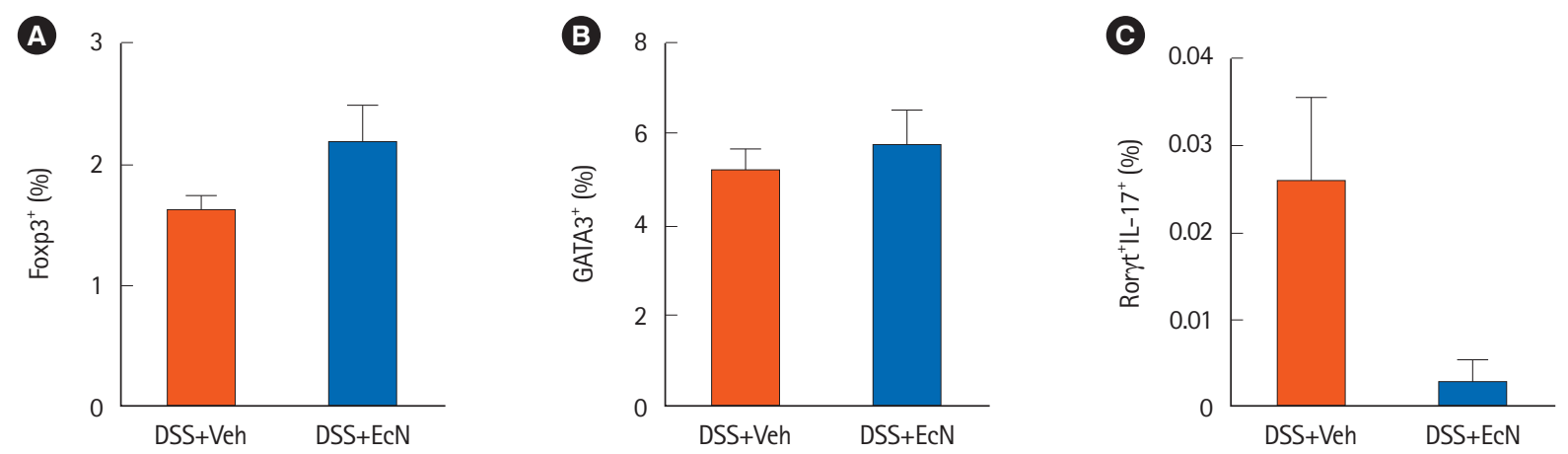

Fig. 2. Anti-inflammatory effects of Escherichia coli Nissle 1917 (ECN) regarding to the inflammatory response associated with T lymphocyte. Splenocytes were isolated from 8-week-old and analyzed T lymphocytes by flow cytometric analysis. Treg differentiation (A), Th1 differentiation (B), and Th17 differentiation (C) in spleens of mice treated with dextran sulfate sodium (DSS) and with or without EcN. Data are expressed as the mean \pm standard error of the mean $(n=4)$. Analyses were performed using Mann-Whitney test. Veh, phosphate buffered saline-treated vehicle. Foxp3, forkhead box P3; GATA3, GATA binding protein 3; Rorrt, retinoic-acid-receptor-related orphan nuclear receptor gamma.

modulate the Treg/Th17 responses to maintain gut homeostasis. Further studies investigating the underlying immunologic mechanisms related protective effects of $\mathrm{EcN}$ on colitis would be warranted. Further studies on the potential of EcN for protective effects against the development of colorectal cancer would also be valuable. ${ }^{10}$ Finally, more clinical data on its clinical relevance are needed to use this probiotics in the treatment of UC.

\section{ADDITIONAL INFORMATION}

\section{Funding Source}

This work was supported by a grant from the Ministry of Health \& Welfare, Republic of Korea (grant number:HI14C1324) and a National Research Foundation of Korea (NRF) grant funded by the Korea government (MSIT) (number: 2020R1A2C2003638).

\section{Conflict of Interest}

Park J and Cheon JH are editorial board members of the journal but were not involved in the peer reviewer selection, evaluation, or decision process of this article. No other potential conflicts of interest relevant to this article were reported.

\section{Author Contribution}

Conceptualization: Kim SW, Cheon JH. Data curation: Park J, Kim DH, Kim S. Formal analysis: Park J, Kim DH, Kim S. Funding acquisition: Cheon JH. Investigation: Park J, Kim DH, Kim S, Ma HW, Park IS, Kim JH, Shin Y, Son M. Methodology: Kim SW, Cheon JH. Project administration: Kim SW, Cheon JH. Resources: Kim SW, Cheon JH. Software: Park J, Kim SW. Supervi- sion: Kim SW, Cheon JH. Validation: Kim SW, Cheon JH. Visualization Park J, Kim SW. Writing - original draft: Park J. Writing - review \& editing: Kim SW, Cheon JH. Approval of final manuscript: all authors.

\section{ORCID}

Park J

Kim DH

Kim S

Ma HW

Park IS

Son $\mathrm{M}$

Kim JH

Shin Y

Kim SW

Cheon JH

https://orcid.org/0000-0002-5836-8735 https://orcid.org/0000-0003-0513-6583 https://orcid.org/0000-0002-2577-2969 https://orcid.org/0000-0002-3891-4495 https://orcid.org/0000-0001-6546-5456 https://orcid.org/0000-0002-5889-8488 https://orcid.org/0000-0001-5381-3628 https://orcid.org/0000-0002-3044-0168 https://orcid.org/0000-0002-1692-1192 https://orcid.org/0000-0002-2282-8904

\section{REFERENCES}

1. Scaldaferri F, Gerardi V, Mangiola F, et al. Role and mechanisms of action of Escherichia coli Nissle 1917 in the maintenance of remission in ulcerative colitis patients: an update. World J Gastroenterol 2016;22:5505-5511.

2. Shafiee NH, Manaf ZA, Mokhtar NM, Raja Ali RA. Anti-inflammatory diet and inflammatory bowel disease: what clinicians and patients should know? Intest Res 2021;19:171-185.

3. Mizoguchi E, Low D, Ezaki Y, Okada T. Recent updates on the basic mechanisms and pathogenesis of inflammatory bowel diseases in experimental animal models. Intest Res 2020;18: $151-167$. 
4. Schultz M, Strauch UG, Linde HJ, et al. Preventive effects of Escherichia coli strain Nissle 1917 on acute and chronic intestinal inflammation in two different murine models of colitis. Clin Diagn Lab Immunol 2004;1 1:372-378.

5. Rodríguez-Nogales A, Algieri F, Garrido-Mesa J, et al. The Administration of Escherichia coli Nissle 1917 ameliorates development of DSS-induced colitis in mice. Front Pharmacol 2018;9:468.

6. Park IS, Son M, Ma HW, et al. Succinate-treated macrophages attenuate dextran sodium sulfate colitis in mice. Intest Res 2021;19:349-353.

7. Sassone-Corsi M, Nuccio SP, Liu H, et al. Microcins mediate competition among Enterobacteriaceae in the inflamed gut. Nature 2016;540:280-283.

8. Kim JM, Cheon JH. Pathogenesis and clinical perspectives of extraintestinal manifestations in inflammatory bowel diseases. Intest Res 2020;18:249-264.

9. Weise C, Zhu Y, Ernst D, Kühl AA, Worm M. Oral administration of Escherichia coli Nissle 1917 prevents allergen-induced dermatitis in mice. Exp Dermatol 2011;20:805-809.

10. Kim SH, Lim YJ. The role of microbiome in colorectal carcinogenesis and its clinical potential as a target for cancer treatment. Intest Res 2021 May 21 [Epub]. https://doi.org/10.5217/ ir.2021.00034. 